Discursos pronunciados en el acto

de transmisión de mando del Instituto

de Estudios Internacionales,

el 4 de agosto de 2014.

\title{
Discurso de despedida del director saliente, Profesor José Morandé Lavín
}

En primer término, deseo felicitar muy sinceramente al Profesor Walter Sánchez González, por su reciente elección como director del Instituto de Estudios Internacionales para el periodo 2014-2018. Hago extensivo este sentimiento a nuestro claustro académico, que en forma decidida y mayoritaria apoyó la postulación del profesor Sánchez.

$\mathrm{Al}$ concluir un periodo de ocho años en la dirección del Instituto de Estudios Internacionales de la Universidad de Chile, quisiera compartir algunas experiencias y reflexiones personales acerca de nuestro legado histórico, identidad y trayectoria institucional reciente.

Desde su creación, el Instituto se ha identificado como un centro de enseñanza e investigación superior sobre los aspectos políticos, jurídicos, económicos e históricos de las relaciones internacionales. Actualmente, lo definimos en nuestro plan de desarrollo institucional como el principal centro académico del país que aborda interdisciplinaria y transversalmente los temas internacionales y que aspira a ser un referente de internacionalización y formación de especialistas para la universidad, para el país, para América Latina y para otras regiones del mundo.

En perspectiva y sin ser historiador de oficio, permítanme algunas licencias para referirme brevemente a nuestra gestión, 
como herederos, testigos y sujetos de nuestro propio pasado, presente y futuro.

Al conmemorar los cuarenta años de vida institucional, nos referíamos al contexto internacional y al ambiente nacional y universitario de mediados de los años sesenta, como también al espíritu imaginativo, creativo y visionario de su fundador Claudio Véliz. En efecto, un conjunto de factores relacionados entre sí, que anticipaba los efectos de una incipiente globalización transnacional en Chile y en la región latinoamericana, hizo propicia la gestación de una idea y la materialización de un proyecto universitario como el del Instituto.

Fue este el escenario que en 1966 llevó a que desde Londres, el profesor Claudio Véliz, con el apoyo entusiasta del reconocido historiador británico Arnold Toynbee y con la colaboración de insignes figuras intelectuales y universitarias de Chile, Europa y América Latina, lideraran la creación de un centro de estudios internacionales en la región.

Bajo la inspiración del Royal Institute of International Affairs o Chatham House, abrieron un espacio desde el cual observar e investigar los nuevos desafíos que presentaba el sistema mundial y, a la vez, facilitar, desde el ámbito académico, propuestas profesionales para la participación de Chile en el concierto internacional.

De esta manera, a partir de la distensión del escenario mundial de la época, junto con la creciente participación de América Latina en la política internacional, las élites políticas y ciudadanas chilenas tuvieron la gran oportunidad de establecer al más alto nivel académico, un centro de investigación, enseñanza y divulgación de los asuntos internacionales dentro de la universidad, fundada y dirigida en los albores de nuestra república por uno de los intelectuales y estadistas más brillantes del siglo XIX, don Andrés Bello.

Así lo entendieron también las autoridades universitarias y de gobierno de la época en nuestro país. En efecto, dos eximios humanistas y educadores públicos chilenos, el rector de la Universidad de Chile, profesor Eugenio González -con el acuerdo del Honorable Consejo Universitario- y el señor ministro de Educación Pública, profesor Juan Gómez Millas, apoyaron y dispusieron en forma entusiasta la creación del 
Instituto de Estudios Internacionales, dependiente de la Rectoría de esta casa de estudios.

Identitariamente, la impronta latinoamericana de esta casa fue definida desde su nacimiento. El instrumento legal respectivo incluyó entre sus objetivos la colaboración científica con la política exterior de los países latinoamericanos y la realización de estudios e investigaciones acerca de los problemas que generan las relaciones internacionales, especialmente en lo que se refiere a sus repercusiones en América Latina.

En virtud de este mandato fundacional, la trayectoria y vigencia actual del Instituto le permite asentar y proyectar su tradición de excelencia académica y ser referente latinoamericano en los estudios internacionales.

Hoy, próximos al medio siglo de existencia, asistimos a una etapa de renovación de la dirección institucional, con un legado de crecimiento académico y con tareas y metas concretas cumplidas y por desarrollar.

Cuando asumimos la dirección del Instituto en 2006, invitamos a los miembros de nuestra comunidad a una tarea conjunta que reflejara ampliamente los valores de la Universidad de Chile, esto es, la apelación a su tradición de tolerancia, diálogo, respeto mutuo y calidad en el trabajo académico. Movidos por este espíritu y con la acogida entusiasta y esperanzadora de muchos, iniciamos una gestión institucional con dedicación y visión universitaria, intentando responder en forma creciente al desafío de actualizar y consolidar la misión rectora de nuestro centro en el campo de los estudios internacionales.

Siempre apostando a la creación de un ambiente de reflexión compartida y de respeto intelectual, nuestra impronta fue salir al encuentro del desafío de promover una gestión directiva con trabajo de equipo y con la participación activa del Consejo del Instituto.

Asimismo, nos propusimos ser un referente en los estudios internacionales en Chile y en la región, lo que actualmente somos en algunos temas. A través de una gestión abierta a nuevas ideas y con el empuje de nuestros académicos y de todos los integrantes de la comunidad del Instituto hemos actualizado y mejorado nuestra llegada a la sociedad chilena. Una sociedad 
llena de nuevos desafíos, que aun hoy y con seguridad mañana, nos seguirá exigiendo cumplir un rol destacado.

\section{Oportunidades docentes:}

En el periodo que concluimos, nuestra prioridad fue ampliar la oferta estudiantil y fortalecer la docencia de calidad. Apostamos en primer término a ampliar y consolidar el posgrado, creando y acreditando un nuevo Magíster Profesional en Estrategia Internacional y Política Comercial. Inaugurado en enero de 2011, contó desde sus inicios con el apoyo de la Organización Mundial del Comercio (OMC) mediante la vinculación efectiva a centros internacionales especializados y de excelencia que operan a través de redes universitarias en Europa y América Latina.

Una de las tres cátedras de la OMC en la región está asentada en Chile y en este Instituto, razón por la cual ha sido posible realizar programas de becas de estudio y desarrollar tareas de docencia, investigación y divulgación adscritas al desarrollo del programa.

Del mismo modo, nuestro Magíster tradicional en Estudios Internacionales obtuvo exitosamente su reacreditación, consolidando un esfuerzo trascendente de 37 años de vigencia y de un reconocido prestigio internacional. El atractivo del programa para estudiantes nacionales y extranjeros es significativamente singular, en especial en el caso de alumnos provenientes de America Latina.

La creación y otorgamiento anual de la Beca de Excelencia Académica profesor José Garrido Rojas a partir del año 2007 ha sido un aliciente necesario, pero no suficiente para estimular y reconocer el mérito académico entre los estudiantes del Magíster en Estudios Internacionales. Debemos continuar promoviendo la realización de mayores esfuerzos para obtener nuevos recursos que permitan aumentar nuestra cobertura de asistencia y becas para los estudiantes de postgrado de ambos programas de Magíster.

Por otra parte y en el marco de una política de educación continua y de requerimientos profesionales y académicos del medio externo, desarrollamos en forma sostenida sendos Diplomas de Postítulo en diferentes materias y áreas profe- 
sionales en el ámbito internacional. Entre ellos cabe destacar los Diplomas en Estudios Internacionales; Política Comercial; Integración Regional; Desarrollo Internacional; Religión y Poder y Conflicto en el Medio Oriente. Los dos últimos, bajo la coordinación y estrecha colaboración con el Centro de Estudios Árabes de la Facultad de Filosofía y Humanidades de la Universidad de Chile.

Mención especial merece el Diploma de Postítulo "La relación chileno-peruana: puntos de encuentro" que venimos realizando desde 2011 en nuestra sede, conjuntamente con la colaboración docente del Instituto de Estudios Internacionales de la Pontificia Universidad Católica del Perú y bajo los auspicios y con el apoyo sostenido y entusiasta de la fundación Konrad Adenauer.

Asimismo, deseo destacar nuestra activa participación docente e institucional en el Diploma en Relaciones Internacionales para alumnos de la Academia Diplomática de Chile Andrés Bello junto con el Instituto de Asuntos Públicos de esta Universidad y la Facultad de Derecho de la Pontificia Universidad Católica de Chile.

Esta última actividad la iniciamos este año a partir de la implementación de una alianza estratégica entre las partes bajo la coordinación del Instituto de Estudios Internacionales y la Academia Diplomática Andrés Bello.

En un futuro cercano y de acuerdo con las nuevas líneas de desarrollo y proyección académica, estaremos en condiciones de impartir programas de postítulo en otras temáticas de las políticas públicas internacionales y áreas regionales, como Bioética y Ética Global y Política internacional de África sub sahariana.

No menos importante para el desarrollo docente y el crecimiento institucional es el proyecto de título profesional de Especialista en Relaciones Internacionales. Al respecto, hemos avanzado de manera significativa en la presentación a las instancias superiores de la Universidad. Tras más de un año de estricta preparación y cumpliendo con acabados estudios, rigor profesional y reglamentario, el proyecto fue aprobado por unanimidad por el Honorable Consejo Universitario y la Comisión Académica del mismo. 
Esperamos que el Senado Universitario se pronuncie favorable y prontamente en pos de esta importante y urgente iniciativa académica.

Finalmente, quisiera resaltar el proyecto conjunto con la Facultad de Filosofía y Humanidades de la Universidad de Chile de crear una Licenciatura en Estudios Internacionales. Los acuerdos logrados y los avances en esta iniciativa docente que compromete al trabajo asociado y transversal de ambas unidades académicas son una apuesta para un trabajo común en el futuro. Nuestra participación institucional y docente nos permitirá aportar las competencias y especialidades de nuestro claustro académico.

Lo anterior nos permitirá también vislumbrar muy pronto la posibilidad de cumplir con un objetivo estratégico, esto es, el de completar paulatinamente el ciclo de formación en Estudios Internacionales, desde el pregrado hasta niveles avanzados en esta área del conocimiento.

Ciertamente que para culminar con el proceso formativo en la materia, la anhelada meta de desarrollar a mediano plazo un programa transversal de doctorado en Estudios Internacionales es posible si cumplimos exitosamente con los estándares y estadios anteriores.

En otras palabras, nuestro gran desafío es perseverar en una fórmula de creación de un programa docente integral que comprenda desde la licenciatura hasta el doctorado. Lo visualizamos como un proyecto transversal con vasos comunicantes con facultades e institutos afines de la Universidad de Chile y con el apoyo de convenios académicos con centros universitarios de excelencia en el extranjero.

\section{Fortalecimiento de la investigación:}

La trayectoria del Instituto nos ha permitido fortalecer y desarrollar lazos con entidades académicas y gubernamentales para generar, entre otros, nuevos conocimientos en el campo de la investigación en asuntos internacionales.

No obstante que en este ámbito es necesario y posible acrecentar los niveles de participación en proyectos concursables en el plano científico nacional e internacional, nuestro interés también se ha expresado en el logro de una valoración de la 
investigación aplicada mediante la generación de respuestas a problemas concretos y propuesta de alternativas frente a las demandas de políticas públicas internacionales.

De esta forma, hemos acogido e impulsado iniciativas en el campo de la política exterior de Chile, así como en cuestiones relacionadas con el desarrollo de negociaciones en el comercio internacional y la inserción económica de Chile y la región en el mundo.

Proyectos como los Estudios de Percepciones de la Opinión Pública en materia de Política Exterior: "Chile, las Américas y el mundo", con el CIDE de México y la AGCI-Chile; "Generacion de diálogo entre Chile-Perú en el marco de los nuevos desafios bilaterales y regionales", con la fundación Adenauer y la Pontificia Universidad Católica del Perú, más los estudios específicos sobre comercio internacional en el marco de la cátedra OMC del Instituto son ejemplo de líneas de trabajo e investigación persistentes y que se proyectan en este ámbito.

Revista Estudios internacionales, biblioteca y digitalización:

Uno de los factores identitarios del instituto nacido simultáneamente con este y que marca su trayectoria y reconocimiento académico es la Revista Estudios Internacionales. De amplio prestigio en América Latina y publicada en forma ininterrumpida desde 1967 (47 años) completó recientemente la edición $\mathrm{N}^{\circ} 178$. A partir del año recién pasado, la revista está indizada en Scielo, objetivo que nos propusimos desde el inicio de nuestra gestión con el respaldo de la dirección de la revista y su comité editorial. Ciertamente ello constituye un logro de excelencia académica e instrumental en el ámbito de las publicaciones, tanto para la Universidad de Chile, como para los investigadores y la comunidad epistémica nacional e internacional respectiva.

Asmismo, el patrimonio académico a nivel nacional e internacional que representa el equipamiento y eficiente funcionamiento de la Biblioteca del Instituto la identifica como la mejor dotada y especializada en su género en Chile y reconocida en Latinoamérica. 
En el transcurso de estos años y en estrecha coordinación y con el apoyo del sistema de bibliotecas de la universidad, hemos digitalizado la Revista Estudios Internacionales desde su primer número publicado en 1967, hasta el más reciente de 2014. En otras palabras, Estudios Internacionales está hoy en línea en el portal de revistas académicas de la Universidad de Chile, con cifras actualizadas de 139.400 visitas el año 2013.

En esta línea de cooperación institucional, en 2009 iniciamos la incorporación de las tesis de Magíster de nuestros alumnos en el Portal de Tesis Electrónicas de la Universidad.

Asimismo, desde 2012 se ha digitalizado la totalidad de la colección de libros del instituto, esfuerzo no menor atendido el numero de volúmenes disponibles en biblioteca.

\section{Extensión, convenios internacionales y difusión:}

Más acotado a la linea de extensión, desarrollamos seminarios, encuentros y mesas redondas a partir de líneas de trabajo propias, en asociación con centros y redes especializadas y con universidades con las cuales tenemos proyectos de cooperación académica.

Bajo esta perspectiva, durante nuestro período firmamos 33 convenios y acuerdos específicos de cooperación con instituciones internacionales, como la Organización Mundial del Comercio, la UNCTAD, la CEPAL, la Fundación Adenauer y FLACSO-Ecuador, entre otras.

También subscribimos acuerdos con unidades académicas asociadas a los estudios internacionales y latinoamericanos de universidades como la Pontificia Universidad Católica de Buenos Aires, la Universidad Nacional de La Plata, la Universidad del Norte, de Barranquilla, la Universidad de Hamburgo y la Universidad de Ankara, Turquía.

Por cierto, junto con constituir una gran oportunidad de desarrollo institucional, esta cooperación nos exige mayores tareas y muchas veces nos vemos sobrepasados por ofertas de colaboración. No obstante, la cooperación nos abre perspectivas reales y muy valiosas para un verdadero desarrollo académico de excelencia.

En consecuencia, todos estos convenios apuntan al desarrollo activo y potencial de la docencia, investigación y difusión 
conjunta en materias afines desarrolladas por las respectivas instituciones.

La difusión de nuestras actividades corporativas y académicas, de acuerdo con los nuevos recursos comunicacionales disponibles en la Universidad y en el Instituto, se ha canalizado a través de una activo y dinámico sitio web. Para estos fines se han creado en el último tiempo dos medios de difusión: Visiones Internacionales y Boletín de Noticias, que permiten la divulgación de estudios, investigaciones, análisis, debates e información académica e institucional relevante.

Del mismo modo, la nueva plataforma institucional en internet incorpora también el uso de redes sociales como Facebook, Linkedin y Twitter, canal a través del cual mantiene una fluida comunicación con alumnos, académicos y entidades, tanto de Chile como del extranjero.

\section{Gestión económica y presupuestaria:}

Retrospectivamente, cuando asumimos la Dirección nos propusimos impulsar la modernización de la gestión del Instituto a través de la profesionalización de las tareas directivas y administrativas, en el marco de un plan de desarrollo institucional.

Concebimos la modernización de la gestión administrativa como la necesidad de contar con recursos humanos dotados de capacidades profesionales y personales que contribuyeran a facilitar una interlocución fluida con la autoridad universitaria competente.

La incorporación de profesionales con conocimientos y experiencia que han permitido optimizar la gestión en las áreas económicas, computación, extensión y difusión del Instituto ha sido ciertamente un logro importante.

En materia de gestión económica y administrativa también ha sido importante la aplicación expedita de políticas modernas en materia de contabilidad, procesos de compra pública, sistemas de información, transparencia, procedimientos y control de gestión desde el nivel central de la Universidad y de los organismos de consultoría externa.

De acuerdo con esta preocupación, nuestra tarea ha sido reconocida y bien evaluada por las instancias correspondien- 
tes. Pese a este reconocimiento, hemos debido enfrentar costos económicos crecientes y restricciones presupuestarias debido a factores propios de las necesidades de desarrollo institucional, así como a las limitaciones estructurales de políticas de financiación universitaria que nos afectan directamente.

A este respecto, abrigo la esperanza de que las políticas anunciadas por la nueva autoridad universitaria tomen en cuenta esta realidad, toda vez que de acuerdo con su origen y legalidad vigente los institutos interdisciplinarios como el nuestro tienen una relación directa con la Rectoría.

Así pues, es necesario y perentorio reconocer que tanto la trayectoria como los desafíos que enfrenta el Instituto de Estudios Internacionales son posibles debido a que es patrimonio de la Universidad de Chile. Nuestra casa de estudios nos ha brindado valiosas oportunidades de investigar, enseñar y difundir al más alto nivel los estudios internacionales en Chile, America Latina y otras latitudes.

\section{Reconocimiento a la autoridad universitaria y agradecimiento a la comunidad del Instituto:}

Sobre este particular deseo expresar nuestro reconocimiento a la autoridad superior de la Universidad que a lo largo de nuestra trayectoria ha manifestado su preocupación y su interés por el instituto y ha valorado las tareas que le son propias.

También debo agradecer el legado de nuestros fundadores y de quienes continuaron su obra como directores y directoras del Instituto, a los cuales he tenido el gran privilegio de conocer y con quienes he compartido personalmente inquietudes, proyectos y sueños institucionales.

Vaya asimismo mi reconocimiento a las entidades extranjeras que contribuyeron y respaldaron al Profesor Véliz en la idea y creación del Instituto. Me refiero a Chattham House, al Centro de Estudios de Política Extranjera de París, y a la London School of Economics and Political Science, entre otros.

Mi personal reconocimiento también a la Rectoría pasada por considerar al Instituto de Estudios Internacionales en los planes de expansión física de la Universidad de Chile y por el apoyo brindado a nuestro traslado a las futuras dependencias 
e instalaciones del proyecto universitario de Avenida Vicuña Mackenna 20.

Mi agredecimiento a toda la comunidad del Instituto, profesores, estudiantes y personal de colaboración, quienes acogiendo los principios y valores de la Universidad de Chile e indentificándose plenamente con nuestro proyecto y misión institucional, entregaron y aportaron esfuerzo, inquietudes, experiencia académica y dedicación profesional durante estos años.

Una tarea común y de equipo es y ha sido esencial para el logro de las metas que nos propusimos en nuestro proyecto de desarrollo institucional. Es por ello que expreso mis agradecimientos al equipo directivo que con plena identificación y compromiso corporativo hizo suyo nuestro mandato y me acompañó incansablemente en esta apuesta universitaria.

Con este espíritu, deseo manifestar mi más sincero reconocimiento y gratitud personal a mi sucesor en la dirección del Instituto de Estudios Internacionales, Profesor Walter Sánchez González. Como director adjunto y director de la Escuela de Graduados del Instituto durante estos ocho años, su contribución leal y entusiasta fue decisiva para el desarrollo de nuestro proyecto institucional y para el logro de los objetivos y metas académicas que nos propusimos.

No me cabe duda alguna de que como director del Instituto de Estudios Internacionales de la Universidad de Chile, el Profesor Sánchez sabrá renovar y proyectar con conocimiento, sabiduría y trabajo de equipo, las motivaciones y aspiraciones de toda la comunidad del Instituto en un nuevo contexto de desafíos y oportunidades a nivel educacional y universitario del país.

Junto con desear el mayor éxito a la nueva Dirección del Instituto, permítanme reiterar mis agradecimientos a la Universidad de Chile y a esta comunidad por haberme brindado la oportunidad y el privilegio de servir, con aciertos y errores, a un grupo humano como el nuestro.

La recreación y desarrollo de un ambiente genuinamente universitario en el Instituto es una tarea permanente y de equipo. Requiere proyectar la trayectoria e identificación institucional sustentada en valores de pluralismo y excelencia 
académica, al mismo tiempo que tolerancia y respeto al mérito y a las personas.

Invocando a estos principios concluyo parafraseando al poeta Benedetti: de no rendirse y retomar el vuelo, pues cada día es un comienzo nuevo. Me asiste la mayor certeza de que el Instituto de Estudios Internacionales con el apoyo de todos sus integrantes continuará el viaje, ya que esta es la hora y el mejor momento.

Muchas gracias 